\title{
Levofloxacin-Induced Tendon Rupture: A Case Report and Review of the Literature
}

\author{
Liana Gold, ARNP, MSN, and Helena Igra, MD
}

Treatment with the fluoroquinolone class of antibiotics has become increasingly popular. Clinician preference for quinolones stems from their excellent gastrointestinal absorption, superior tissue penetration and broad-spectrum activity. ${ }^{1,2}$ However, this has led to widespread and indiscriminate use, affecting microbial resistance patterns and increasing drug-related morbidity. ${ }^{3}$ Although quinolone-induced tendonopathy and tendon rupture have been previously described in the literature, reports of tendon rupture in association with newer quinolones such as levofloxacin are now emerging. ${ }^{4,5}$ We describe a patient with levofloxacininduced partial rupture of the Achilles tendon and review the literature, pathophysiology, predisposing factors, and treatment recommendations.

\section{Case Report}

An 88-year-old man was admitted with a recurrent draining abdominal abscess. A dye study confirmed an enterocutaneous fistula for which a right colon resection was performed. Past medical history included hypothyroidism, parkinsonism, dementia, osteoporosis, and prostate cancer previously treated with leuprolide. Medications at admission included levothyroxine, carbidopa/levodopa, donepezil, alendronate sodium, calcium, fludrocortisone, baby aspirin, and vitamin $\mathrm{E}$.

Oral levofloxacin, $500 \mathrm{mg} /$ day, was initiated 5 days before admission. After surgery, the patient received $500 \mathrm{mg}$ of intravenous levofloxacin daily with cefazolin for 5 days and then was continued on daily oral levofloxacin at the same dosage for an additional 5 days.

Discomfort in the left ankle was noted 2 days after the initial dose of levofloxacin and progressed

Submitted, revised, 13 February 2003.

From the Departments of Internal Medicine (LG) and Dermatology (HI), Mount Sinai Medical Center, Miami Beach, Florida, and Private Practice of Dermatology, Miami Beach, Florida. Address correspondence to Helena Igra, MD, 4045 Sheridan Ave, \#253, Miami Beach, FL 33140 (e-mail: drhelena@the-beach.net). throughout the peri- and post-operative period. One week after discharge, he presented to the emergency department with mild, nonpitting edema of the left calf and ankle, and pain on plantar flexion. No Homan sign could be elicited. A Doppler study failed to detect a thrombus, and there was no radiologic evidence of a fracture. The patient was diagnosed with a muscle strain and discharged from the hospital with a prescription for $100 \mathrm{mg}$ of celecoxib twice per day for pain and $20 \mathrm{mg}$ of furosemide per day for edema.

Two weeks later, he was re-evaluated in the emergency department because of severe left leg pain, increased edema, erythema, and warmth extending to the knee, with tenderness on palpation of the Achilles tendon. A second Doppler study was negative, and the patient was admitted for a presumed cellulitis that resolved rapidly on intravenous cefazolin. However, he continued to complain of ankle pain on weight bearing.

We suspected a quinolone-induced tendonopathy, so magnetic resonance imaging (MRI) was performed, which confirmed a 2-cm segment of partial tearing of the Achilles tendon, approximately $4 \mathrm{~cm}$ above the calcaneal insertion with surrounding soft tissue edema (Figure 1). Because the patient was not a good surgical candidate, a below-the-knee ankle-foot orthosis was applied for 3 months. He received regular physical therapy while remaining non-weight-bearing at home. Full recovery ensued without any sequelae of tendonopathy.

\section{Discussion}

Although quinolone-induced tendon rupture has been described in the literature, ${ }^{6,7}$ case reports of complete or partial tendon rupture specifically attributed to levofloxacin are rare thus far. ${ }^{4,5}$ Quinolone-induced tendonopathy was noted as early as 1983; Bailey et $\mathrm{al}^{8}$ reported a norfloxacin-related tendonitis in a patient with kidney failure. The first case of a tendon rupture associated with ciprofloxacin was reported in 1987. ${ }^{9}$ A pefloxacin-related 


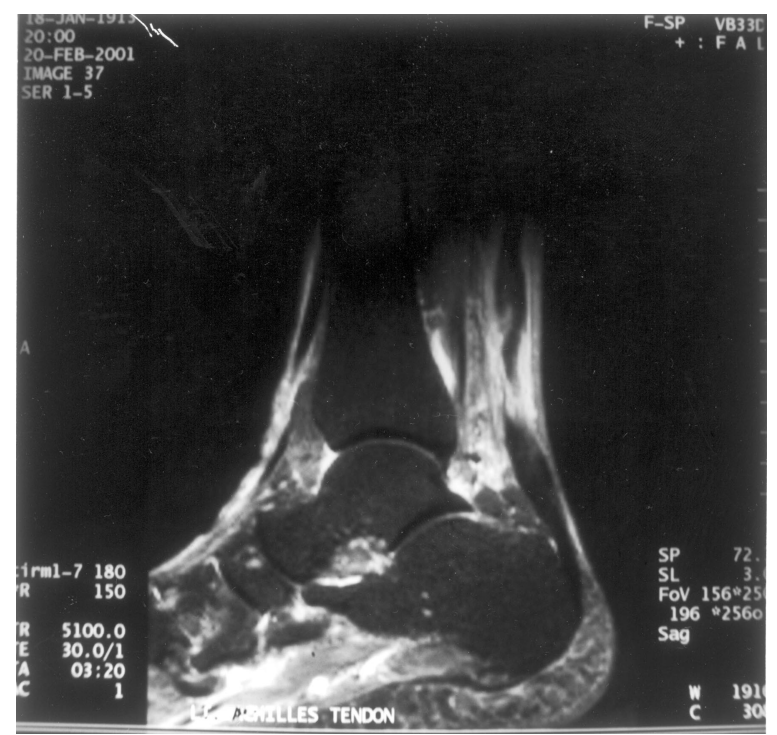

Figure 1. MRI of a 2-cm segment of an Achilles tendon partial tear, $4 \mathrm{~cm}$ above the calcaneal insertion with surrounding soft tissue edema.

tendon rupture reported in 1991 led to the published recognition of this complication in the VIDAL (French PDR) in 1992. In October 1996, the US Food and Drug Administration first issued a Report of Adverse Events and revised the class labeling for fluoroquinolones to include a warning of possible tendonitis/tendon rupture after more than 200 reports of quinolone-related tendonopathy over a 10 -year period. ${ }^{10} \mathrm{~A}$ warning based on postmarketing surveillance reports was added to the PDR in December 2001, indicating that this risk may be increased in patients receiving concomitant corticosteroids, especially in the elderly. ${ }^{2}$ An epidemiologic study from the United Kingdom in 2002 calculated the excess risk for quinolone-induced tendonopathy at 3.2 per 1000 patient years. ${ }^{11}$ A 2001 worldwide surveillance report estimated levofloxacin-associated tendon ruptures at less than 4 per million prescriptions. ${ }^{5}$

The presentation of quinolone-induced tendonitis is characteristically abrupt in onset, with sharp pain occurring spontaneously with walking and/or palpation. ${ }^{10}$ Although most tendon ruptures occur after 2 weeks, they can occur as early as a few hours after the initial dose or up to 6 months after drug therapy. A direct relationship exists between severity and the length of treatment; there is a predilection for the Achilles tendon, but shoulder and hand involvement has been reported as well., ${ }^{2,6,12}$ The injury may be bilateral, partial, or complete and is usually located 2 to $3 \mathrm{~cm}$ above a poorly vascularized area. ${ }^{1,13}$ On examination, substantial swelling is frequently noted, and the presentation may mimic phlebitis. The patient usually presents with a positive Thompson sign (absence of plantar flexion on squeezing the calf muscle in the prone position). ${ }^{1,10}$ Pain diminishes after tendon rupture occurs. The clinical diagnosis may be confirmed by ultrasound or more precisely by MRI. An MRI of a new rupture shows evidence of edema, hemorrhage and fiber discontinuity. ${ }^{1,7,10}$

Treatment consists of immediate discontinuation of quinolone therapy at the earliest suspicion of tendonopathy. ${ }^{7,12}$ For mild tendonitis, weightbearing restriction ranges from 2 to 6 weeks. Tendon rupture, whether surgically or conservatively treated, requires casting and prolonged rest. The duration of immobilization varies from 6 weeks to 6 months. ${ }^{1,10}$ This can be especially debilitating to the elderly, more so if bilateral injury is involved. Thirty percent of patients proceed to tendon rupture despite adequate intervention. Once a quinolone-induced tendonopathy is suspected, a patient should not be rechallenged with quinolones. ${ }^{10}$

The pathologic mechanisms responsible for quinolone-induced tendonopathy seem to be multifactorial. Studies have implicated ischemic, toxic, and matrix-degrading processes. Jorgensen et $\mathrm{al}^{14}$ described degenerative tendon lesions with interstitial edema and necrosis. Quinolone-induced tendon rupture more often occurs in less vascularized areas, which further supports an ischemic process. ${ }^{13}$ Premarketing studies found quinoloneinduced macroscopic cartilaginous erosive lesions in weight-bearing joints of juvenile dogs, leading to the early contraindication for quinolone use during childhood, adolescence, pregnancy, and nursing. ${ }^{2,10}$ In an in vitro study, exposure of tendon tissue to ciprofloxacin showed a $60 \%$ to $68 \%$ decrease in fibroblast proliferation, a $36 \%$ to $48 \%$ decrease in collagen synthesis, a $14 \%$ to $60 \%$ decrease in proteoglycans synthesis, and a significant increase in matrix-degrading proteolytic activity after only 72 hours in culture. ${ }^{15}$ Reports of tendonopathy occurring hours after a single dose further suggest direct cytotoxicity. ${ }^{10,16}$

Risk factors for tendonitis/tendon rupture are emerging from postmarketing surveillance. The most commonly reported risk factors are concomitant steroid therapy and renal insufficiency. ${ }^{4,10,11,17}$ Other conditions that may predispose a 
patient to a quinolone-related tendon rupture include advanced age, prior tendonopathy, magnesium deficiency, hyperparathyroidism, diuretic use, peripheral vascular disease, rheumatoid arthritis, diabetes mellitus, and strenuous sports activities. ${ }^{1,11,18}$ The patient in this case report noted symptoms of tendonitis within days after oral levofloxacin was initiated. MRI confirmed the diagnosis of tendon rupture 3 weeks later. His risk factors included advanced age, steroid therapy, a brief course of diuretics, and prolonged exposure to levofloxacin.

\section{Conclusion}

The deleterious effects of fluoroquinolones on tendons have been documented since the 1980s. A concomitant rise in tendon rupture incidence has been observed during this same time span. ${ }^{19,20}$ With the increasing use of levofloxacin and other quinolone antibiotics, we should expect to encounter a growing number of patients experiencing tendonopathy. Patients presenting with joint tenderness and swelling, especially those in a high-risk category, should be questioned as to quinolone use dating as far back as 6 months or more. Discontinuation of the medication and immobilization of the affected joint should be foremost. Preventative measures include avoiding indiscriminate use of quinolones, recognition of risk factors, and adherence to renal dosing. Finally, an emphasis on patient awareness can further reduce the morbidity associated with quinolone-induced tendonitis and/or rupture by prompting earlier presentation, evaluation, and intervention.

We are grateful to Michael Gold, MD, and Luis Gruberg, MD, for their editorial assistance.

\section{References}

1. Zabraniecki L, Negrier I, Vergne P, et al. Fluoroquinolone induced tendinopathy: report of 6 cases. J Rheumatol 1996;23:516-20.

2. Physicians' desk reference. Montvale, NJ: Medical Economics Co, 56th ed, 2002. Levaquin, p. 253743.

3. Davidson R, Cavalcanti R, Brunton JL, et al. Resistance to levofloxacin and failure of treatment of pneumococcal pneumonia. N Engl J Med 2002;346: 747-50.

4. Weller S. Minerva. BMJ 2002;324:554.
5. Kahn JB. Latest industry information on the safety profile of levofloxacin in the US. Chemotherapy 2001;47 Suppl 3:32-7; discussion 44-8.

6. Casparian JM, Luchi M, Moffat RE, Hinthorn D. Quinolones and tendon ruptures. South Med J 2000; 93:488-91.

7. McGarvey WC, Singh D, Trevino SG. Partial Achilles tendon ruptures associated with fluoroquinolone antibiotics: a case report and literature review. Foot Ankle Int 1996;8:496-8.

8. Bailey RR, Kirk JA, Peddie BA. Norfloxacin-induced rheumatic disease [letter]. N Z Med J 1983;96:590.

9. McEwan SR, Davey PG. Ciprofloxacin and tenosynovitis [letter]. Lancet 1988;2:900.

10. Harrell RM. Fluoroquinolone-induced tendinopathy: what do we know? South Med J 1999;92:622-5.

11. van der Linden PD, Sturkenboom MC, Herings RM, Leufkens HG, Stricker BH. Fluoroquinolones and risk of Achilles tendon disorders: case-control study. BMJ 2002;324:1306-7.

12. Gabutti L, Stoller R, Marti HP. Fluoroquinolones as etiology of tendinopathy. Ther Umsch 1998;55: 558-61.

13. Huston KA. Achilles tendinitis and tendon rupture due to fluoroquinolone antibiotics [letter]. N Engl J Med 1994;331:748.

14. Jorgensen C, Anaya JM, Didry C, et al. Arthropathy with Achilles tendon involvement induced by pefloxacin. Apropos of a case. Rev Rhum Mal Osteoartic 1991;58:623-5.

15. Williams RJ, Attia E, Wickiewicz TL, Hannafin JA. The effect of ciprofloxacin on tendon, paratenon, and capsular fibroblast metabolism. Am J Sports Med 2000;28:364-9.

16. Bernard-Beaubois K, Hecquet C, Hayem G, Rat $P$, Adolphe M. In vitro study of cytotoxicity of quinolones on rabbit tenocytes. Cell Biol Toxicol 1998;14: 283-92.

17. Medwatch. Summary of safety-related drug labeling changes approved by FDA center for drug evaluation and research (CDER). Levaquin. Washington, DC: United States Food and Drug Administration; 2001 Dec. Available at: URL: http://www.fda.gov/ medwatch/safety/2001/dec01.htm\#levaqu

18. Shakibaei M, de Souza P, van Sickle D, Stahlmann R. Biochemical changes in Achilles tendon from juvenile dogs after treatment with ciprofloxacin or feeding a magnesium-deficient diet. Arch Toxicol 2001;75:369-74.

19. Leppilahti J, Puranen J, Orava S. Incidence of Achilles tendon rupture. Acta Orthop Scand 1996;67: 277-9.

20. Maffulli N, Waterston SW, Squair J, Reaper J, Douglas AS. Changing incidence of Achilles tendon rupture in Scotland: a 15-year study. Clin J Sport Med 1999;9:157-60. 